\title{
Acesso e regulação ao cuidado especializado no Rio Grande do Sul: a estratégia RegulaSUS do TelessaúdeRS-UFRGS
}

\author{
Access and regulation of specialized care in Rio Grande do Sul: \\ the RegulaSUS strategy of TelessaúdeRS-UFRGS
}

\author{
Natan Katz (https://orcid.org/0000-0002-0659-7747) ${ }^{1}$ \\ Rudi Roman (https://orcid.org/0000-0002-2663-4314) ${ }^{1}$ \\ Dimitris Varvaki Rados (https://orcid.org/0000-0003-0819-5343) ${ }^{1}$ \\ Elise Botteselle de Oliveira (https://orcid.org/0000-0001-9552-3282) ${ }^{1}$ \\ Carlos André Aita Schmitz (https://orcid.org/0000-0002-9003-9704) ${ }^{2}$ \\ Marcelo Rodrigues Gonçalves (https://orcid.org/0000-0001-8516-8547) ${ }^{2}$ \\ Sotero Serrate Mengue (https://orcid.org/0000-0002-3349-8541) ${ }^{2}$ \\ Roberto Nunes Umpierre (https://orcid.org/0000-0001-9841-5543) ${ }^{2}$
}

${ }^{1}$ Núcleo Técnico-Científico de Telessaúde, Universidade Federal do Rio Grande do Sul. R. Mostardeiro 366/901 Moinhos de Vento. 90430000 Porto Alegre RS Brasil. natankatzmed@gmail.com

${ }^{2}$ Universidade Federal do

Rio Grande do Sul. Porto Alegre RS Brasil.

\begin{abstract}
In Primary Health Care (PHC), access, and integrality are strongly influenced by the coordination of care, which in turn receives a positive impact from the articulation of telehealth actions for teleregulation of care. We created a teleregulation method (RegulaSUS Project) based on specific protocols firmly grounded in scientific evidence. From data of the regulatory system and TelessaúdeRS, we explored the effects of RegulaSUS on PHC and access to specialized care. This method set comprehensive protocols, with a significant mean reduction of $30 \%$ in the specialized visits queue over 360 days. It reduced waiting time for medical clinical visits (median of 66 days) but not for surgical appointments. Waiting times for queued cases varied inversely, increasing for clinical and declining for surgical specialties. The use of teleconsultations unrelated to regulation increased with the exposure of professionals to RegulaSUS. The intervention evidence potentiality in the integration of health systems, mainly among low-and middle-income countries, and makes telehealth act as a meta-service, building efficient, qualified, and equitable networks.
\end{abstract}

Key words Primary Health Care, Telemedicine, Teleregulation, Outpatient care, Rio Grande do Sul
Resumo Na Atenção Primária à Saúde (APS) acesso e integralidade são fortemente influenciados pela coordenação do cuidado, que por sua vez recebe impacto positivo da articulação de ações de telessaúde para a telerregulação da assistência. Criamos uma metodologia de telerregulação (Projeto RegulaSUS) baseada em protocolos específicos firmemente alicerçados em evidências. A partir de dados do sistema de regulação e do TelessaúdeRS exploramos os efeitos do RegulaSUS na APS e no acesso ao cuidado especializado. A metodologia foi capaz de criar protocolos abrangentes, com expressiva redução média da fila de consultas especializadas de 30\% em 360 dias. Reduziu o tempo de espera na marcação de consultas em especialidades clínicas (mediana de 66 dias), mas não em cirúrgicas. Tempos de espera nos casos mantidos em fila variaram de forma inversa, aumentado em especialidades clínicas e diminuindo em cirúrgicas. O uso de teleconsultorias espontâneas aumentou com a exposição dos profissionais ao RegulaSUS. A intervenção tem potencial na integração de sistemas de saúde, principalmente em países de baixa e média renda, e faz com que a telessaúde atue como metasserviço, construindo redes eficientes, qualificadas e equânimes.

Palavras-chave Atenção Primária à Saúde, Telemedicina, Telerregulação, Assistência ambulatorial, Rio Grande do Sul 


\section{Introdução}

O acesso é um desafio ubíquo e deficiências na porta de entrada prejudicam a consecução dos demais atributos essenciais da Atenção Primária à Saúde (APS) ${ }^{1,2}$. A partir da década de 80 , o Brasil passou por uma ampliação exponencial da $\mathrm{APS}^{3}$, que não foi espelhada na atenção ambulatorial, um vazio demográfico profissional onde apenas $4,8 \%$ dos médicos do país atuam ${ }^{4}$. Da mesma forma, a incorporação tecnológica atingida pela atenção hospitalar não foi estendida aos demais níveis. Com isso, temos um cenário de uma APS heterogênea, com dificuldades para impedir que condições de saúde sensíveis a sua atuação contribuam para a lotação tanto das listas de espera por atenção ambulatorial, como das unidades de pronto atendimento ${ }^{5-7}$. É inefetivo aumentar o acesso pela ampliação isolada da oferta (Lei de Roemer $)^{8}$ sem passar pela qualificação da oferta e pela organização da demanda, ambas situações sensíveis a ações de Telessaúde9. Assim como no Sistema Único de Saúde (SUS) não existe universalidade e integralidade sem equidade (e não há equidade sem regulação do acesso à assistência), na APS o acesso e a integralidade são fortemente influenciados pela coordenação do cuidado ${ }^{1,10}$.

O Núcleo de Telessaúde do Rio Grande do Sul (TelessaúdeRS), que está oficialmente registrado no Cadastro Nacional de Estabelecimentos de Saúde (CNES) do Brasil, faz parte do Programa de Pós-Graduação em Epidemiologia da Faculdade de Medicina da Universidade Federal do Rio Grande do Sul (UFRGS) e recebe apoio e recursos do Ministério da Saúde (MS) e da Secretaria Estadual de Saúde do Rio Grande do Sul (SES-RS). Com um grupo, inicialmente sem grande domínio ou fluência tecnológica, mas com grande naturalidade para transitar pela Epidemiologia e pela APS, o TelessaúdeRS iniciou suas atividades em novembro de 2007. Partimos de uma visão de futuro de atendimento em saúde com mínimas barreiras e com os pés firmes em um pensamento científico e na aplicação do melhor conhecimento disponível. Como tínhamos à mão poucas ferramentas tecnológicas, iniciamos em 2007 as primeiras teleconsultorias utilizando apenas editores de texto e servidores de e-mail, em formato assíncrono?.

Uma década depois, apesar da telepresença tornar-se uma realidade no dia a dia do TelessaúdeRS (mais de 20 mil pessoas avaliadas por teleoftalmologia a partir de 2017), a tecnologia continua tendo um papel coadjuvante, pois um dos grandes diferenciais do núcleo é prover teleassistência baseada em evidências atuais e robustas para toda a APS brasileira. Em 2013, para tomar impulso para esse salto a frente, foi necessário dar um passo atrás e, contrapondo a corrente de incorporação de tecnologias cada vez mais complexas, adotar uma tecnologia do milênio passado: a telefonia convencional ${ }^{9}$. Por meio de uma linha telefônica gratuita 08006446543 conseguimos atingir todos os estados brasileiros chegando a mais de 145 mil teleconsultorias telefônicas respondidas em aproximadamente seis anos, de forma síncrona e sem custo para os solicitantes. O TelessaúdeRS também provê cobertura em espirometria, dermatologia e estomatologia para todo o Estado (mais de 40 mil laudos) e ações de tele-educação para todo o país (mais de 40 mil profissionais certificados em cursos EAD, com cerca de três milhões de visualizações do conteúdo online e mais de 700 mil downloads dos 22 aplicativos produzidos) ${ }^{11}$.

A regulação de encaminhamentos da APS para a atenção especializada é um problema ao mesmo tempo local e mundial. Envelhecimento da população, avanços no conhecimento biomédico, aumento da prevalência das condições de saúde e critérios diagnósticos progressivamente mais estritos levaram a aumento das taxas de multimorbidade $^{12}$. Dessa forma, é esperado que encaminhamentos para atenção especializada aumentem $^{13,14}$. Nos Estados Unidos da América a taxa de que uma consulta ambulatorial leve a encaminhamento aumentou de 4,8\% para 9,3\% no período de 1999 a $2009^{15}$. Encaminhamentos adequados aumentam a qualidade do cuidado, apesar disso, a alta variabilidade dos encaminhamentos e ausência de parâmetros claros e taxas aceitáveis de encaminhamentos sugerem que tanto uso excessivo e deficiente da atenção especializada ocorrem ${ }^{16-18}$. Além disso, a alta demanda por encaminhamentos aumenta a taxa de espera para consulta especializada ${ }^{19}$.

O desenvolvimento de mecanismos para coordenação do cuidado pela APS está associado com melhora dos desfechos na transição do cuidado para a atenção especializada ${ }^{20}$. Por meio do papel de coordenação e ordenação da rede consegue-se expansão dos cuidados clínicos e aumento da resolubilidade da APS, portanto diminuindo o risco de investigações e tratamentos excessivos ${ }^{21}$. Mais importante, isso pode aumentar o acesso dos pacientes ao médico generalista e estimular esse vínculo. Além disso, é possível otimizar o uso de recursos, evitar viagens desnecessárias e aumentar a eficiência e equidade na gestão das listas de espera, obtendo-se definições objetivas para encaminhamento de cada condição de saúde ${ }^{22,23}$. 
Entretanto, para esse papel de coordenação e para um uso otimizado da APS, são necessárias informações mínimas sobre os pacientes para definição da indicação e da prioridade para o atendimento especializado ${ }^{16,24-27}$. Neste contexto, protocolos de encaminhamentos podem guiar os atendimentos da APS e auxiliam o papel de coordenação e ordenamento do cuidado.

Apesar disso, o simples desenvolvimento de protocolos de encaminhamentos não garante a qualidade da informação, uma vez que a adesão dos profissionais a esses protocolos costuma ser baixa $^{28}$. Ações regulatórias devem ser implementadas para garantir que todo o potencial da APS seja utilizado antes do encaminhamento. Sistemas de saúde e redes de atendimento são bastante diversos em termos de tamanho, estrutura e financiamento. O Brasil tem dimensões continentais e o atendimento especializado é ofertado em uma ou poucas cidades maiores em cada estado. Isso leva a viagens frequentes e longas para consultas presenciais e comunicação difícil entre médicos realizando os atendimentos na atenção primária à saúde (sejam Médicos de Família e Comunidade ou não) e especialistas. Essa configuração cria uma oportunidade para integrar ações de telessaúde e regulação ao mesmo tempo que otimiza a resolubilidade da APS.

Já nos primórdios das teleconsultorias do TelessaúdeRS observamos que elas têm a capacidade de evitar aproximadamente $60 \%$ de encaminhamentos para atenção especializada. Com esse efeito a integração das ações de telessaúde com o processo de regulação de pedidos de encaminhamentos para atenção especializada parecia um passo natural ${ }^{9}$. Além da teleconsultoria, a telerregulação usa de forma aditiva outras ações de Telessaúde (telediagnóstico, tele-educação) e o TelessaúdeRS, por sua vez, usa a telessaúde não como um serviço de saúde, mas como um metasserviço, capaz de fortalecer linhas reguladas de união entre os pontos assistenciais ${ }^{29}$.

Considerando esse contexto, de impacto na coordenação do cuidado por meio da articulação de ações de telessaúde para a telerregulação da assistência, desenvolvemos uma metodologia de trabalho capaz de atingir dois objetivos simultâneos: (1) auxiliar os processos de regulação de consultas especializadas do interior para a capital do estado; (2) ampliar a eficácia e o acesso na APS através do uso de telerregulação. Apresentamos neste trabalho a metodologia desenvolvida no projeto RegulaSUS e os resultados alcançados pelo TelessaúdeRS - UFRGS.

\section{Breve caracterização do Estado do Rio Grande do Sul}

O Estado do Rio Grande do Sul, localizado no extremo sul do Brasil, tem população estimada de 11,4 milhões de habitantes distribuída em 497 municípios e $281,7 \mathrm{mil} \mathrm{km}^{2}$. Tem por limites Santa Catarina, outro estado brasileiro, Argentina e Uruguai além do Oceano Atlântico. O último Índice de Desenvolvimento Humano (IDH) do Estado é 0,746. Contava com 2.701 estabelecimentos de saúde da rede pública e 3.004 da rede privada no ano de 2009. A agropecuária e a indústria representam as principais economias.

\section{Metodologia}

O Projeto RegulaSUS e as ações de telerregulação tiveram origem em 2012 por meio de uma aproximação entre a academia e a gestão pública, ainda como um projeto piloto, culminando em 2014 com um convênio entre Secretaria Estadual de Saúde do Rio Grande do Sul e TelessaúdeRS.

\section{Desenvolvimento de protocolos}

Como passo inicial para atingir os objetivos foi necessário o desenvolvimento de protocolos de encaminhamento. O conjunto de informações clínicas necessárias para o encaminhamento deve ser sucinto, objetivo e limitado ao necessário para a tomada de decisão. $\mathrm{Na}$ busca de um método para desenvolvimento de protocolos de encaminhamento da APS para a atenção especializada, nós desenvolvemos um passo a passo para criação (ou adaptação) local de protocolos. Esse processo segue sete passos a seguir especificados:

1. Identificação de uma especialidade ou procedimento que tenha demanda reprimida (medida por tempos de espera prolongados) e que os motivos de encaminhamento possam ser manejados na APS. Como piloto para desenvolver essa estratégia optamos por abordar a fila de endocrinologia, que apresentava uma fila de espera de mais de oito mil pessoas e tempos de espera maiores de um ano;

2. Avaliar a demanda dentro da fila de espera escolhida. Nós selecionamos uma amostra de 5\% da fila, estratificada por mês, para superar eventuais fenômenos sazonais. Depois avaliamos as informações subjetivas de cada referenciamento. Apesar da disponibilidade do Código Internacional de Doenças, ele não foi utilizado por não ser confiável; 
3. Selecionar as condições de encaminhamento mais frequentes para aquela especialidade/procedimento. Essas condições devem cobrir 80-85\% de todos os encaminhamentos;

4. Revisar os protocolos clínicos e evidências científicas disponíveis sobre o manejo clínico das condições selecionadas. As referências incluem livros texto de APS, diretrizes e ferramentas de suporte à decisão médica. Tomamos especial atenção para esgotar as referências de APS e atentamos para as contribuições da atenção especializada no manejo das condições/doenças selecionadas;

5. Definir para cada protocolo as situações que devem e não devem ser encaminhadas para avaliação especializada, criando assim os gatilhos para encaminhamento da APS para a atenção especializada. No nosso método, cada protocolo foi construído por um Médico de Família e Comunidade e revisado por outros dois generalistas (Médicos de Família e Comunidade ou Internistas). Por fim, os protocolos foram discutidos com especialistas vinculados a um hospital universitário;

6. Determinar quais situações apresentam maior risco e, portanto, devem ter acesso facilitado, considerando o melhor interesse do paciente. Em situações de tempos de espera prolongados, essas condições devem ter baixa prevalência (não devem representar mais que $10 \%$ da demanda para especialidade). Na nossa amostra inicial, Diabetes Mellitus tipo 1, hipertireoidismo e alta suspeita para neoplasia de tireoide foram consideradas prioritárias para avaliação presencial com endocrinologista;

7. Estabelecer as informações mínimas a serem fornecidas pelos médicos da APS. Essas informações devem ser dados da história clínica e exame físico, e exames complementares disponíveis na APS. Portanto, essas informações devem ser suficientes para estabelecer o diagnóstico ou suspeita clínica e guiar os próximos passos do médico regulador (conforme definido pelo protocolo). Esses dados devem ser objetivos, limitados a cinco a oito parâmetros por condição de saúde (Quadro 1).

Seguindo esse processo, nós desenvolvemos protocolos para cada uma das condições de saúde. Por exemplo, para hipertireoidismo cinco parâmetros são questionados: sinais e sintomas, nível de TSH, nível de T4, uso de antitireoidianos e uso de outras medicações. Dados sobre idade e sexo já são fornecidos automaticamente pelo sistema de regulação. Essas informações permitem o médico regulador inferir se o paciente tem de fato hipertireoidismo, se está sintomático e se o paciente já está em tratamento. Uma tarefa importante na criação do protocolo é remover qualquer questionamento não essencial para o processo de regulação. Protocolos ágeis são mais amigáveis e convenientes, características essenciais para a prática da APS.

\section{Implementação e integração com as ações de telessaúde}

Após o desenvolvimento, os protocolos foram aprovados pela Secretaria Estadual de Saúde do Rio Grande do Sul. Todos os pedidos de consulta com atenção especializada são analisados por médicos reguladores (Figura 1). Apesar do desenvolvimento dos protocolos, os encaminhamentos são realizados de forma não estruturada (texto livre) pelos médicos da APS. Utilizando os protocolos, os reguladores podiam autorizar o encaminhamento (quando informações fossem suficientes), solicitar novas informações para o médico da APS ou município ou indicar uma teleconsultoria telefônica com um médico do TelessaúdeRS - UFRGS, bem como lançar mão de outras ações de telessaúde. Os teleconsultores são Médicos de Família e Comunidade ou especialistas focais que trabalham no Canal 0800 do TelessaúdeRS, atuando de forma individual ou em interconsultoria. As teleconsultorias não eram compulsórias, mas as informações deveriam ser informadas por telefone ou pelo sistema de encaminhamento eletrônico. Os teleconsultores recebem suporte de um grupo de monitores, que prepara as discussões e uma amostra das discussões passa por auditoria. O fluxo da Figura 1, especialmente modelado em BPMN (Business Process Model and Notation $)^{30}$ para esse trabalho, bem como os fluxos de teleconsultoria, telediagnóstico e Canal 0800 foram registrados no Instituto Nacional de Produção Industrial.

\section{Análise dos dados}

Avaliamos a eficácia do método em identificar os principais motivos de encaminhamento (passo 2). Para isso, na endocrinologia, avaliamos o percentual de cada motivo selecionado na amostragem inicial com os motivos de encaminhamento identificados no período de novembro de 2013 a maio de 2016.

Para analisar os efeitos do processo de trabalho desenvolvido e implementado utilizamos o banco de dados do sistema informatizado de encaminhamentos AGHOS. Na presente análise 
Quadro 1. Passo a passo para criação/adaptação local de protocolos utilizados pelo Projeto RegulaSUS, TelessaúdeRS-UFRGS, 2019.

1. Identificar especialidades com demanda reprimida e motivos de encaminhamento sensíveis à atenção primária à saúde;

2. Amostrar a demanda reprimida;

3. Estabelecer os motivos mais frequentes de encaminhamento;

4. Revisar a literatura correspondente aos motivos frequentes;

5.Definir, para cada motivo de encaminhamento, quais situações clínicas justificam atenção especializada;

6. Determinar as situações clínicas prioritárias para atendimento especializado;

7. Estabelecer o conjunto mínimo de informações necessárias para o encaminhamento.

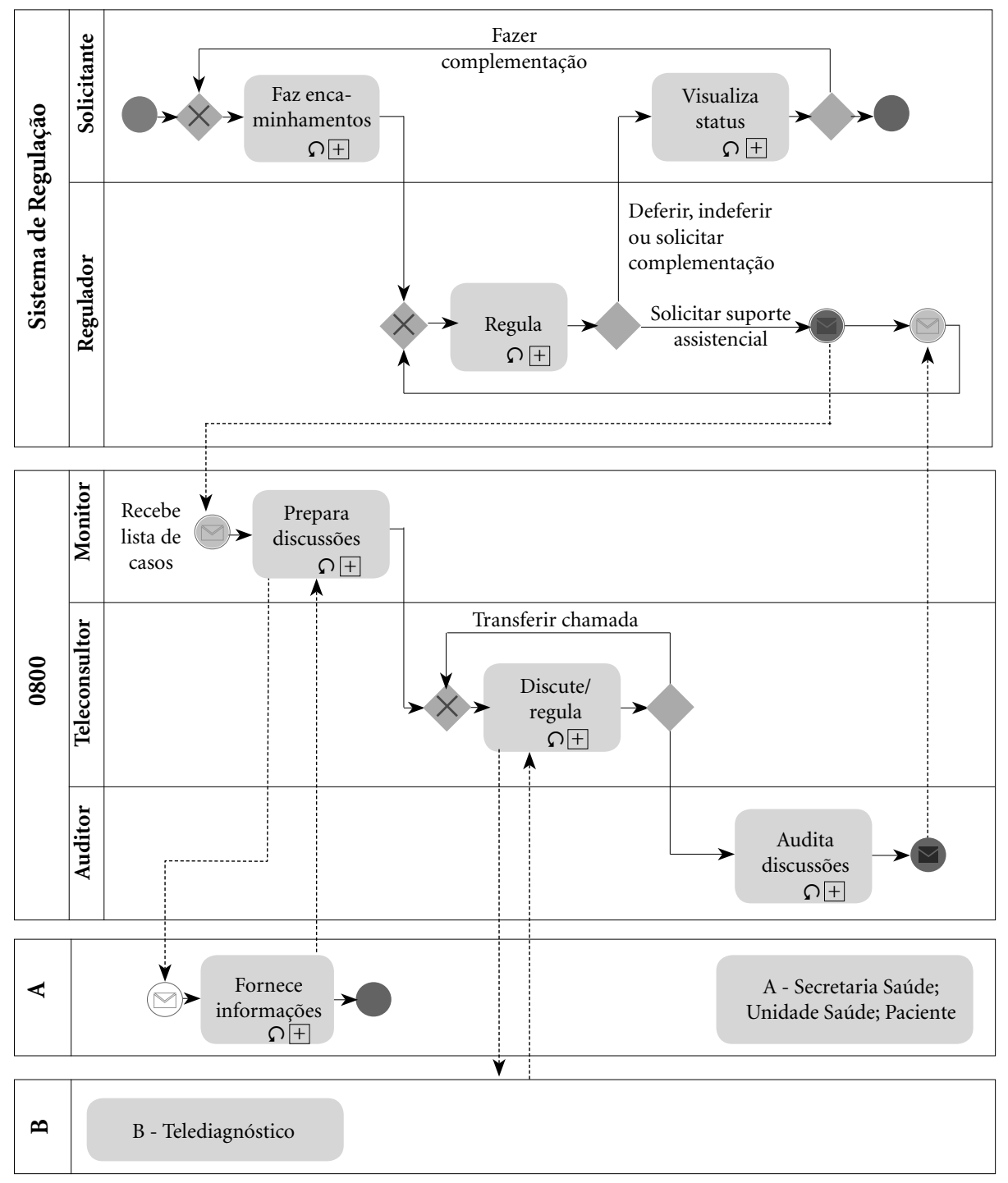

Figura 1. Modelagem do fluxo de solicitação de ações de telessaúde como suporte à Central de Regulação Ambulatorial do Estado. TelessaúdeRS-UFRGS, Porto Alegre, 2015. 
incluímos os encaminhamentos das filas acompanhadas pelo RegulaSUS por pelo menos um ano até a inativação do sistema. O AGHOS foi inativado e substituído por um novo sistema em junho de 2016. Com esse banco, fizemos uma análise retrospectiva das seguintes variáveis: 1) tamanho da fila e; 2) tempo de espera (mediana) dos encaminhamentos não agendados em cada momento do tempo (utilizamos o início da aplicação dos protocolos como tempo zero e observamos a situação da fila 90, 60 e 30 dias antes até 30, 60, 90, 180 e 360 dias após); 3) número de marcações ocorridas nos 30 dias anteriores a cada momento do tempo e; 4) tempo de espera (mediana) até essa marcação. Os resultados foram analisados agregados (totais e categorizados em especialidades clínicas e cirúrgicas) e divididos por especialidades.

Além disso, buscamos dados de uso do canal de teleconsultorias e avaliamos os efeitos das consultorias do RegulaSUS no uso espontâneo do Canal 0800.

\section{Resultados}

\section{Implementação e acurácia dos protocolos}

Os protocolos de encaminhamento foram implementados ao longo do ano de 2015 para a regulação de toda a fila de especialidades selecionadas. As filas a seguir foram incluídas no RegulaSUS, portanto nessa análise, de acordo com a seguinte cronologia: endocrinologia (01/01/2015); nefrologia (01/01/15); pneumologia (19/02/15); urologia (19/02/15); neurocirur-

Tabela 1. Proporção de condições de saúde selecionadas amostradas e encontradas na população. TelessaúdeRS-UFRGS, Porto Alegre, 2019.

\begin{tabular}{lcc}
\hline \multicolumn{1}{c}{ Condição de saúde } & Amostra & População \\
\hline Diabetes Mellitus & $30 \%$ & $20,3 \%$ \\
Nódulos de tireóide & $26 \%$ & $34,6 \%{ }^{*}$ \\
Hipotireoidismo & $20 \%$ & $15,0 \%$ \\
Hipertireoidismo & $6 \%$ & $7,0 \%$ \\
Bócio & $6 \%$ & $34,6 \%{ }^{*}$ \\
Obesidade & $5 \%$ & $6,8 \%$ \\
Outras (condições não & $7 \%$ & $16,3 \%$ \\
protocolizadas) & & \\
Total & $100 \%$ & $100,0 \%$ \\
\hline
\end{tabular}

* as condições de bócio e nódulos foram agregadas na análise final dos dados gia (15/05/15); neurologia (15/05/15); cirurgia torácica (15/06/15); reumatologia (15/06/15).

$\mathrm{Na}$ amostra realizada dos encaminhamentos de endocrinologia foram selecionadas seis condições de saúde. Após o desenvolvimento dos protocolos, mais de nove mil encaminhamentos dessa especialidade foram regulados. A amostragem se mostrou capaz de prever as condições mais frequentes de encaminhamento na amostra completa (Tabela 1).

\section{Efeitos na fila de espera}

A implementação dos protocolos integrada com o processo de telerregulação está associada, do ponto de vista cronológico, com uma redução geral do volume da fila de espera por consulta especializada de aproximadamente 30\% (37.435 para 26.172), ocorrendo de maneira mais expressiva a partir de 180 - 360 dias do início da intervenção e nas especialidades clínicas (Tabela 2).

Quanto aos tempos de espera para marcação, observamos que, mesmo ocorrendo uma redução de $27 \%$ na oferta de marcações de consultas (clínicas e cirúrgicas), houve redução de 66 dias na mediana de tempo de espera para marcação de especialidades clínicas sob intervenção (de 234 para 168). Em contrapartida, nas especialidades cirúrgicas a tendência foi inversa, com aumento da mediana de tempo de espera (de 219 para 376).

Outra medida de interesse são as medianas de tempo de espera dos casos que estão na fila de espera a cada momento do tempo (Gráfico 1). Nesse caso, observa-se dilatação do tempo mediano para as especialidades clínicas (de 328 para 361) e redução para as especialidades cirúrgicas (de 302 para 261), ao contrário das tendências dos tempos para marcação.

\section{Efeito nas teleconsultorias espontâneas}

Com o crescimento das discussões de casos em fila de espera, estimulada pela exposição ao RegulaSUS, observa-se um aumento de $860 \%$ associado ao número de teleconsultorias espontâneas (não relacionadas com regulação) realizadas pelo TelesssaúdeRS (Gráfico 2) de jan/14 a jun/16. Observa-se uma inflexão no número de teleconsultorias em fev/16. Esse achado não tem explicação clara nos nossos dados, mas é potencialmente atribuível a um mês com menor número de dias úteis. 


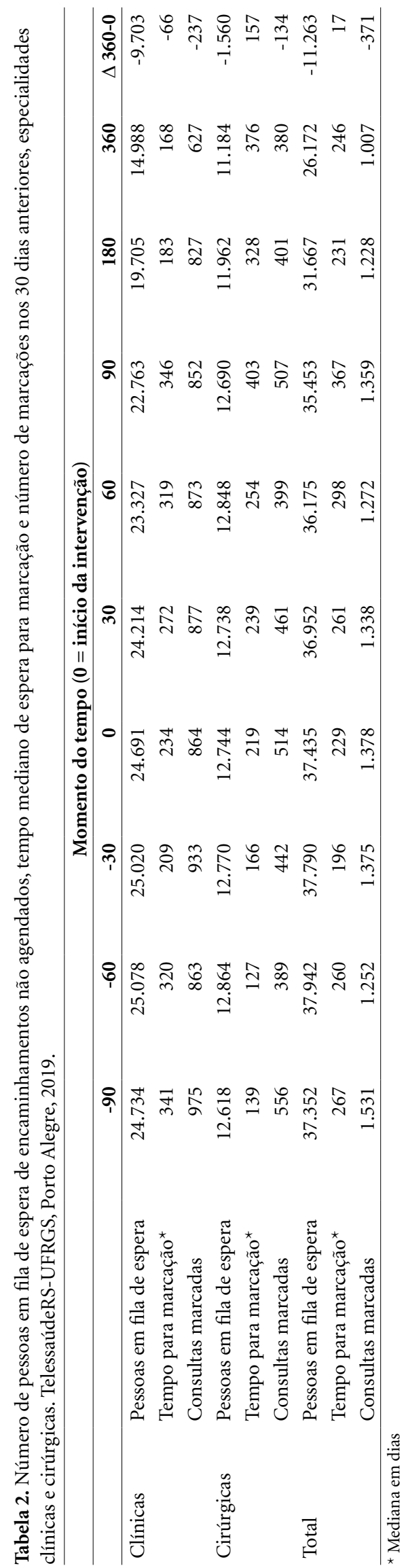

\section{Discussão}

As ações de telessaúde, coordenadas pelo RegulaSUS, foram responsáveis pela redução nas filas de espera das especialidades médicas estudadas, principalmente nas clínicas, sendo que o período em que o impacto demonstrou maior força foi a partir dos 180 dias de início da atuação nas listas. Além destes resultados, o estímulo ao uso espontâneo do Canal 0800 pelos médicos qualifica o cuidado, amplia a capacidade resolutiva da APS e o acesso à atenção especializada na própria APS, além de permitir o uso mais racional da oferta já reduzida de consultas disponíveis no Estado. A hipótese de aumento das consultorias espontâneas do 0800 induzido pelo RegulaSUS é corroborada por estudo prévio que encontrou uma média de teleconsultorias para profissionais que utilizam somente o Canal 0800 de 6,52, enquanto que essa média foi de 10,03 entre aqueles expostos ao RegulaSUS ${ }^{31}$.

O impacto na redução das filas e no tempo mediano de espera para marcação atingido nas especialidades clínicas reflete o potencial que ações de suporte assistencial não presencial, tais como teleconsultoria e telediagnóstico, têm sobre condições sensíveis à telessaúde, mesmo em situações adversas (redução de mais de 1/4 da oferta de consultas especializadas ao longo do período) como demonstradas em nosso estudo. O papel desenvolvido pela telerregulação neste projeto foi de explorar alternativas às consultas especializadas presenciais para os casos com demandas de baixa densidade tecnológica. Esse mesmo aspecto do trabalho explica as limitações da intervenção nas especialidades cirúrgicas, observada a partir de tempos de espera crescentes para marcação de consulta presencial (Tabela 2). A dilatação dos tempos nas especialidades cirúrgicas denota insuficiência de oferta que piora ao longo dos meses. Nessas especialidades, apesar de menor impacto da intervenção do que nas especialidades clínicas, o papel da telerregulação por meio da aplicação dos protocolos e discussão dos casos em teleconsultoria, pode ter atenuado tendências ainda maiores de restrição do acesso e assegurado que muitos casos legados que se avolumavam na fila fossem qualificados para marcação. Como consequência observamos diminuição do tempo de espera dos casos que restam em fila nas especialidades cirúrgicas (Gráfico 1).

Um aspecto importante de inovação presente neste estudo está no desenvolvimento de protocolos de encaminhamento simplificados capazes de abarcar os motivos de encaminhamento mais 


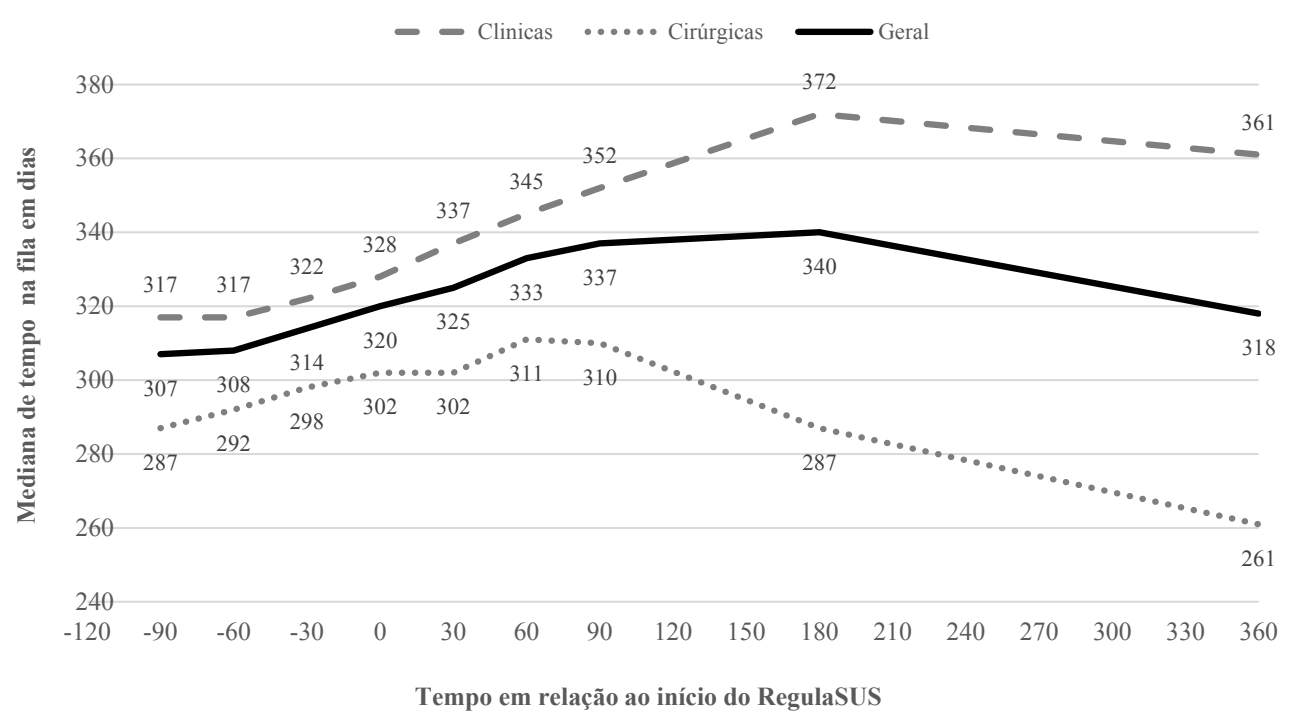

Gráfico 1. Tempo em fila de espera para consulta especializada.

Nota: Mediana de dias em fila de espera das solicitações do interior do estado do RS para consulta presencial na capital nas especialidades sob intervenção do RegulaSUS. Tempo zero representa o início da intervenção. Fonte: TelessaúdeRS-UFRGS e sistema AGHOS, Porto Alegre, 2019.

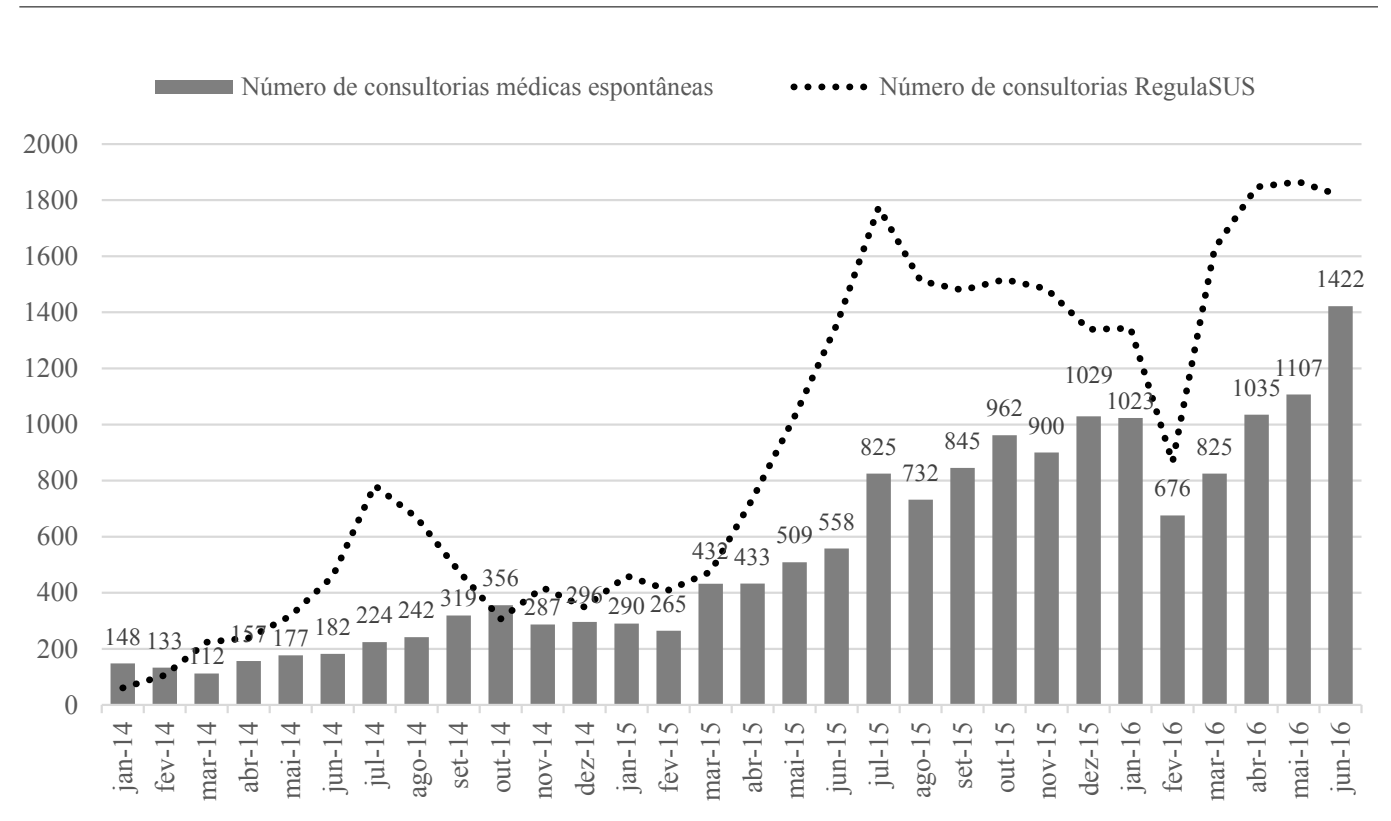

Gráfico 2. Teleconsultorias médicas espontâneas e do RegulaSUS no Rio Grande do Sul.

Nota: Número de teleconsultorias realizadas no serviço do TelessaúdeRS-UFRGS no período compreendido entre janeiro/14 e junho/16.

Fonte: TelessaúdeRS-UFRGS, Porto Alegre, 2019. 
frequentes. De maneira geral (e com alguma evidência) sugere-se como padrão-ouro protocolos e diretrizes amplos e muitas vezes complexos no seu manuseio ${ }^{32,33}$. Entretanto, a utilização, usabilidade e satisfação dos profissionais com tais protocolos estão associados com facilidade de uso e incorporação nos sistemas eletrônicos de consulta $^{25,34-36}$. O papel de liderança dos médicos de APS na confecção de tais materiais foi essencial, contribuindo com seu maior conhecimento sobre as limitações do escopo da prática clínica neste nível de atenção, além do entendimento sobre a função ordenadora da APS no sistema de saúde. A revisão pelos médicos especialistas focais, assim como pelos médicos reguladores, foi igualmente importante para adicionar aspectos relevantes aos protocolos e validar com a prática no mundo real.

É inequívoco que os sistemas de saúde precisam ampliar sua capacidade resolutiva com soluções alternativas ao cuidado tradicional especialmente em tempos de crises financeiras e austeridade. A pressão econômica, tanto pelo aumento da demanda quanto pela incorporação de novas tecnologias, exige soluções criativas orientadas para otimizar acesso, qualidade e custo, pois ações de telessaúde são capazes de atenuar o problema do triângulo de ferro da saúde ${ }^{37,38}$. Nesse sentido, protocolos de encaminhamento simplificados, objetivos e oficiais, mais que meros documentos de gaveta, tem o potencial de estabelecer papéis claros e transparentes dos diferentes níveis de atenção dentro do sistema de saúde e que se contrapõem a prática clínica totalmente autônoma, muito danosa no contexto de recursos limitados. Algo que, apesar de simples, tem pouquíssimos e pontuais precedentes no Brasil.
Consultas eletrônicas como estratégia de aumento do acesso à atenção especializada na APS têm sido adotadas em diferentes partes do mundo e para diferentes especialidades médicas ${ }^{39}$. Em que pese a maioria dessas iniciativas explorarem formatos assíncronos, o modelo desenvolvido no RegulaSUS explora a interação síncrona telefônica, muitas vezes realizada na presença do próprio paciente, o que pode otimizar o número de encontros com o paciente e o cuidado imediato.

Entre as principais limitações encontradas no presente estudo podemos citar o desenho de quase-experimento, com caráter descritivo das ações e dos resultados, sem controle para potenciais fatores confundidores e vieses. Os dados utilizados provêm de uma base secundária e estão sujeitos a problemas de preenchimento e resgate de informações. As medidas de acesso avaliadas - saída da fila por exposição ao RegulaSUS e tempos de espera para marcação - são agregadas e indiretas, não considerando os desfechos em nível individual. Da mesma maneira, não cabe inferência causal do crescimento das consultorias espontâneas associado a evolução da telerregulação uma vez que não foram explorados dados individuais.

Por fim, entendemos que a autonomia médica em processos tais como de encaminhamentos deve ser substituída por estratégias de regulação baseadas em coordenação do cuidado pela APS (gatekeeping), preferencialmente através de ações de telessaúde, garantindo o encaminhamento ao especialista certo, no tempo certo e no lugar certo ${ }^{40}$. Estas ações têm um potencial papel na integração dos sistemas de saúde, principalmente entre os países de baixa e média renda, atuando como metasserviço ${ }^{29}$ e construindo redes eficientes, qualificadas e equânimes.

\section{Colaboradores}

N Katz realizou a concepção do estudo, coleta e interpretação dos dados, revisão de literatura e redação da versão inicial do manuscrito. DV Rados e R Roman participaram do delineamento, coleta, análise e interpretação dos dados, revisão da literatura e versão final do manuscrito. EB Oliveira participou da coleta, análise e interpretação dos dados, e revisão crítica. CAA Schmitz, MR Gonçalves, SS Mengue e RN Umpierre participaram da análise e interpretação dos dados, e revisão crítica. Todos os autores participaram da redação (em seus estágios iniciais ou final) e aprovaram a versão final do manuscrito. 


\section{Agradecimentos}

Gostaríamos de agradecer a colaboração de Alfredo Montelongo Flores pela heurística aplicada ao banco de dados do sistema de regulação que permitiu a reconstrução da fila nos diferentes momentos do tempo.

\section{Referências}

1. Kringos DS, Boerma WGW, Hutchinson A, van der Zee J, Groenewegen PP. The breadth of primary care: a systematic literature review of its core dimensions. BMC Health Serv Res 2010; 10:65.

2. Wakeman SE, Barnett ML. Primary Care and the Opioid-Overdose Crisis - Buprenorphine Myths and Realities. N Engl J Med 2018; 379(1):1-4.

3. Mendes EV. O cuidado das condições crônicas na atenção primária à saúde. Rev Bras Promoç Saude 2018; 31(2):1-3.

4. Scheffer M, coordenador. Demografia Médica no Brasil 2015. São Paulo: Departamento de Medicina Preventiva da Faculdade de Medicina da USP, Conselho Regional de Medicina do Estado de São Paulo, Conselho Federal de Medicina; 2015.

5. Chomatas E, Vigo A, Marty I, Hauser L, Harzheim E. Avaliação da presença e extensão dos atributos da atenção primária em Curitiba. Rev Bras Med Fam Comunidade 2013; 8(29):294-303.

6. Gonçalves MR, Hauser L, Prestes IV, Schmidt MI, Duncan BB, Harzheim E. Primary health care quality and hospitalizations for ambulatory care sensitive conditions in the public health system in Porto Alegre, Brazil. Fam Pract 2016; 33(3):238-242.

7. Harzheim E. Panorama tecnológico da área de telemedicina do complexo da saúde. Brasília: Agência Brasileira de Desenvolvimento Industrial; 2015.

8. Roemer MI. Bed supply and hospital utilization: a natural experiment. Hospitals 1961; 35:36-42.

9. Harzheim E, Gonçalves MR, Umpierre RN, Siqueira ACS, Katz N, Agostinho MR, Oliveira EB, Basso J, Roman R, Dal Moro RG, Pilz C, Heinzelmann RS, Schmitz CA, Hauser L, Mengue SS. Telehealth in Rio Grande do Sul, Brazil: Bridging the Gaps. Telemed J E Health 2016; 22(11):938-944.

10. Mendes EV. As redes de atenção à saúde: revisão bibliográfica, fundamentos, conceito e elementos constitutivos. Brasília: OPAS; 2011.

11. Núcleo de Telessaúde da Universidade Federal do Rio Grande do Sul (TelessaúdeRS). Institucional Curriculum 2007-2018 [documento na Internet]. Disponível em: https://www.ufrgs.br/telessauders/cv

12. Starfield B. Challenges to primary care from co- and multi-morbidity. Prim Health Care Res Dev 2011; 12(1):1-2.

13. Forrest CB, Nutting PA, von Schrader S, Rohde C, Starfield B. Primary care physician specialty referral decision making: patient, physician, and health care system determinants. Med Decis Making 2006; 26(1):76-85.

14. Shea D, Stuart B, Vasey J, Nag S. Medicare physician referral patterns. Health Serv Res 1999; 34(1 pt 2):331348.

15. Barnett ML, Song Z, Landon BE. Trends in physician referrals in the United States, 1999-2009. Arch Intern Med 2012; 172(2):163-170.

16. Mehrotra A, Forrest CB, Lin CY. Dropping the baton: specialty referrals in the United States. Milbank $Q$ 2011; 89(1):39-68.

17. Davies P, Pool R, Smelt G. What do we actually know about the referral process? Br J Gen Pract 2011; 61(593):752-753. 
18. Macinko J, Starfield B, Shi L. Quantifying the Health Benefits of Primary Care Physician Supply in the United States. Int J Health Serv 2007; 37(1):111-126.

19. Stainkey LA, Seidl IA, Johnson AJ, Tulloch GE, Pain T. The challenge of long waiting lists: how we implemented a GP referral system for non-urgent specialist appointments at an Australian public hospital. BMC Health Serv Res 2010; 10:303.

20. Mariotti G, Gentilini M, Dapor V. Improving referral activity on primary-secondary care interface using an electronic decision support system. Int J Med Inform 2013; 82(12):1144-1151.

21. Jamoulle M, Roland M. Champs d'action, gestion de l'information et formes de prévention clinique en médecine générale et de famille. Santé Conjuguée 2005; 33:71-77.

22. Silva SF. Organização de redes regionalizadas e integradas de atenção à saúde: desafios do Sistema Único de Saúde (Brasil). Cien Saude Colet 2011; 16(6):27532762.

23. Chen AH, Murphy EJ, Yee HF. eReferral - A New Model for Integrated Care. N Engl J Med 2013; 368(26):2450-2453.

24. Bouamrane MM, Mair FS. A qualitative evaluation of general practitioners' views on protocol-driven eReferral in Scotland. BMC Med Inform Decis Mak 2014; 14:30.

25. Esquivel A, Sittig DF, Murphy DR, Singh H. Improving the effectiveness of electronic health record-based referral processes. BMC Med Inform Decis Mak 2012; 12:107.

26. Berta W, Barnsley J, Bloom J, Cockerill R, Davis D, Jaakkimainen L, Mior AM, Talbot Y, Vayda E. Enhancing continuity of information: essential components of consultation reports. Can Fam Physician 2009; 55(6):624-5.e1-5.

27. Molero JM, Pérez Morales D, Brenes Bermúdez FJ, Naval Pulido E, Fernández-Pro A, Martín JA, Castiñeiras Fernández J, Cozar Olmo JM. Criterios de derivación en hiperplasia benigna de próstata para atención primaria. Aten Primaria 2010; 42(1):36-46.

28. Kennedy A-M, Aziz A, Khalid S, Hurman D. Do GP referral guidelines really work? Audit of an electronic urgent referral system for suspected head and neck cancer. Eur Arch Otorhinolaryngol 2012; 269(5):15091512.

29. Harzheim E, Chueiri PS, Umpierre RN, Gonçalves MR, Siqueira ACS, D’Avila OP, Bastos CGM, Katz N, Dal Moro RG, Telles LF, Schmitz CAA. Telessaúde como eixo organizacional dos sistemas universais de saúde do século XXI. Rev Bras Med Fam Comunidade 2019; 14(41):1881.

30. International Organization for Standardization (ISO). Information technology - Object Management Group Business Process Model and Notation. Genebra: ISSO; 2013.

31. Rodrigues ÁS. Impacto das teleconsultorias recebidas via projeto RegulaSUS na quantidade de teleconsultorias solicitadas via canal 0800 [trabalho de conclusão de curso]. Porto Alegre: Universidade Federal do Rio Grande do Sul; 2016.
32. Berta W, Barnsley J, Bloom J, Cockerill R, Davis D, Jaakkimainen L, Mior AM, Talbot Y, Vayda E. Enhancing continuity of information: essential components of a referral document. Can Fam Physician 2008; 54(10):1432-1433.

33. Hartveit M, Thorsen O, Biringer E, Vanhaecht K, Carlsen B, Aslaksen A. Recommended content of referral letters from general practitioners to specialised mental health care: a qualitative multi-perspective study. BMC Health Serv Res 2013; 13:329.

34. Thorsen O, Hartveit M, Baerheim A. The consultants' role in the referring process with general practitioners: partners or adjudicators? a qualitative study. BMC Fam Pract 2013; 14:153.

35. Rowe AK, Savigny D, Lanata CF, Victora CG. How can we achieve and maintain high-quality performance of health workers in low-resource settings? Lancet 2005; 366(9490):1026-1035.

36. Kim Y, Chen AH, Keith E, Yee HF, Kushel MB. Not Perfect, but Better: Primary Care Providers' Experiences with Electronic Referrals in a Safety Net Health System. J Gen Intern Med 2009; 24(5):614-619.

37. Kissick WL. Medicine's dilemmas: infinite needs versus finite resources. New Haven: Yale University Press; 1994.

38. Bashshur RL, Howell JD, Krupinski EA, Harms KM, Bashshur N, Doarn CR. The Empirical Foundations of Telemedicine Interventions in Primary Care. Telemed J E Health 2016; 22(5):342-375.

39. Liddy C, Moroz I, Mihan A, Nawar N, Keely EA. Systematic Review of Asynchronous, Provider-to-Provider, Electronic Consultation Services to Improve Access to Specialty Care Available Worldwide. Telemed J E Health 2019; 25(3):184-198.

40. Kirsh SR, Ho PM, Aron DC. Providing specialty consultant expertise to primary care: an expanding spectrum of modalities. Mayo Clin Proc 2014; 89(10):1416-1426

Artigo apresentado em 02/10/2019

Aprovado em 23/10/2019

Versão final apresentada em 25/10/2019 
